\title{
VARIABILIDADE ESPACIAL E TEMPORAL DA RESISTÊ NCIA MECÂNICA DO SOLO À PENETRAÇÃO EM ÁREAS COM E SEM MANEJ O QUÍMICO LOCALIZADO(1)
}

\author{
E. MERCANTE (2), M. A. URIBE-OPAZO(3) \& E. G. SOUZA ${ }^{(4)}$
}

\begin{abstract}
RESUMO
O objetivo deste trabalho foi avaliar o comportamento das variabilidades (espacial e temporal) por meio da técnica de escalonamento de semivariogramas, da variável resistência mecânica do solo à penetração (RMSP) nas camadas de 0-0,1, 0,1-0,2, 0,2-0,3 m, para os anos de 1999 a 2001, sob duas formas de manejo: com manejo químico localizado (CML) e sem manejo químico localizado (SML). Os resultados demonstraram que a variável RMSP apresentou variabilidade espacial com comportamento distinto, conforme a camada e o ano de estudo. A variável RMSP apresentou variabilidade temporal tanto nas parcelas CML quanto nas parcelas SML. As duas formas de manejo (com manejo químico localizado (CML) e sem manejo químico localizado (SML)) não influenciaram o comportamento espacial da variável resistência mecânica do solo à penetração (R MSP). O escalonamento dos semivariogramas reduzi u o tempo computacional de ajuste dos modelos, não apresentando diferenças no comportamento e amplitude da variabilidade espacial em relação aos semivariogramas não escalonados.
\end{abstract}

Termos de indexação: geoestatística, variabilidade espacial e temporal, escalonamento de semivariogramas.

(1) Parte dos Resultados da Dissertação de Mestrado do primeiro autor, apresentada ao Curso de Engenharia Agrícola da Universidade Estadual do Oeste do Paraná -UNIOESTE. Recebido para publicação em janeiro de 2003 e aprovado em outubro de 2003.

(2) Engenheiro Agrícola, pesquisador do Núcleo de Inovações Tecnológicas, Universidade Estadual do Oeste do Paraná - NIT/ UNIOESTE (PR). R. Universitária 2069, CEP 85814-110 Cascavel (PR). Bolsista-CAPES. E-mail: erivelto.mercante@agr.unicamp.br

(3) Professor Adjunto do Centro de Ciências Exatas e Tecnológicas e pesquisadoras do Núcleo de Inovações Tecnológicas, NIT/ UNIOESTE. E-mail: mopazo@unioeste.br

(4) Professor Associado do Centro de Ciências Exatas e Tecnológicas e pesquisadoras do Núcleo de Inovações Tecnológicas, NIT/ UNIOESTE. E-mail: godoy@unioeste.br 


\title{
SUMMARY: SPATIAL AND TEMPORAL VARIABILITY OF THE SOIL MECHANICAL RESISTANCE TO PENETRATION IN AREAS WITH AND WITHOUT SITE-SPECIFIC CHEMICAL APPLICATION
}

\begin{abstract}
The objective of this work was to evaluate the behavior of the spatial and temporal variabilities with the technique of rescaling semi variograms. Study variable was the soil mechanical resistance to penetration (SMRP) in the layers 0-0.1, 0.1-0.2, 0.2-0.3 $\mathrm{m}$ for the years of 1999 to 2001, under two management systems: with (WSS) and without si tespecific nutrient management (NoSS). Results demonstrated that the variable SMRP presented spatial variability with different behavior, according to the soil layer and study year. The variable SMRP presented temporal variability in both management systems. These two systems did not influence the space behavior of the study variable (RMSP). Reescaling of the semivariograms reduced the computing time of model adjustment, and did not present differences in behavior or range of the space variability compared to the not rescaled semivariograms.
\end{abstract}

Index terms: geoestatistics, spatial and temporal variabilities, rescaled semivariograms.

\section{INTRODUÇÃO}

A agricultura convencional, praticada pela maioria dos agricultores, geralmente faz uso maciço de insumos agrícolas. Estes, além de aumentar os custos de produção, podem causar contaminação das águas superficiais e subterrâneas, comprometendo, assim, a utilização deste recurso natural, imprescindível à humanidade. Os compromissos assumidos no desenvol vimento sustentável obrigam a adoção de tecnologias avançadas, com o objetivo de diminuir as diferenças entre a produtividade experimental ereal, não obstanteas particularidades dos diferentes agroecossistemas (Manzatto et al., 2000).

Agricultura de precisão é um conceito relativamente recente de manejo de solo-plantaatmosfera, baseado em princípios de gerenciamento agrícola de informações sobre as variabilidades (espacial e temporal) dos fatores de produção e da própria produtividade. A adoção desta nova tecnologia justifica-se pelo fato de a maioria dos produtores agrícol as considerar o solo uniforme para cada área de cultivo. Porém, cada área pode ter consideráveis variações em seus atributos, tais como: variabilidade espacial do tipo de solo, da produtividade, das características físicas e da necessidade de nutrientes. Para estudar a variabilidade espacial desses atributos, utilizam-se técnicas como a geoestatística, baseada na "teoria das variáveis regionalizadas".

Conhecer a variabilidade espacial dos atributos do sol o que controlam a produtividade das culturas é um fator indispensável à instalação de um programa deagricultura de precisão. Existem várias técni cas disponíveis para conhecer tal variabilidade, tais como: levantamento dos solos, amostragem do solo, utilização de sensores, técnicas de sensoriamento remoto ou, ainda, utilização de model os de simulação (Corá \& Marques J r., 1998).

A variabilidade espacial dos solos não tem sido devidamentetomada em consideração nos processos produtivos da agricultura. Um maior conhecimento da variabilidade espacial da produtividade e das propriedades físicas do solo, bem como o estudo desta variabilidade com o passar dos anos (variabilidade temporal), vem somar com o conhecimento já existentesobre o assunto, o qual, aoser devidamente disponi bilizado aos produtores que adotam a técnica de agricultura de precisão, pode viabilizar uma agricultura moderna, economicamente competitiva e ecol ogicamente correta.

A resistência mecânica do solo à penetração é uma propriedade física relativamente fácil de ser obtida e, de certa forma, de ser correlacionada com a densidade e com a macroporosidade. Para um mesmo solo, quanto maior for a densidade do solo, maior será a resistência à penetração e menor será a macroporosidade, que é o principal espaço para o crescimento das raízes. Deve ser levado em conta, no entanto, que a resistência do solo é mais afetada pela variação nos conteúdos de umidade do solo no momento da amostragem do que pela densidade do solo. Por esta razão, muitos dos trabalhos que objetivam caracterizar o efeito da compactação sobre o desenvol vimento radicular esobrea produtividade das culturas utilizam a densidade do solo como parâmetro de referência (Torres \& Saraiva, 1999).

Para Klein et al. (1998), a resistência mecânica do sol oà penetraçãoéinfluenciada por vários fatores, sendo a densidade e a umidade os principais. Os autores citam que, em uma pesquisa de resistência mecânica do solo à penetração, é fundamental que se faça o monitoramento dos dados de densidade e 
de umidade, uma vez que esses fatores influenciam diretamente os valores da resistência mecânica do solo à penetração.

Torres \& Saraiva (1999) descrevem que valores em torno de 2,5 MPa são considerados baixos, ao passo que valores em torno de 3,5 a 6,5 MPa, aparentemente, são considerados como valores capazes de causar problemas de impedimento mecânico para o desenvolvimento radicular das plantas. Devem-seconsiderar as resistências emtorno de 3,5 MPa, como indicativo de baixa compactação, e de 6,5 MPa, como indicativo de alta compactação.

N este contexto, o objetivo do trabal ho foi estudar o comportamento da variabilidade, espacial e temporal, da resistência mecânica do solo à penetração nas camadas de 0-0,1, 0,1-0,2 e 0,2-0,3 m, nos anos de 1999 a 2001, sob duas formas de manejo: com manejo quími co localizado (CML) esem manejo químico localizado (SML).

\section{MATERIAL E MÉTODOS}

A pesquisa foi realizada em uma área pertencente ao Centro de Pesquisa Eloy Gomes da Cooperativa Central Agropecuária de Desenvolvimento Tecnológico e Econômico Ltda (COODETEC), situada na BR 467, km 98, elocalizada na cidade de Cascavel, Paraná (longitude 53 26 ' W e latitude 24 56 'S). A pesquisa encontra-se em desenvolvimento desde 1998 pelo Núcleo de Inovações Tecnológicas (NIT), da Universidade Estadual do Oeste do Paraná (UNIOESTE).

O sol o foi classificado como Latossolo Vermel ho distroférrico típico, conforme EMBRAPA (1999). O clima da região apresenta-se como temperado mesotérmico e superúmido, tipo climático Cfa (Köeppen) (CASCAVEL, 1995).

Primeiramente, na área experimental, foram demarcadas 256 parcelas, seguindo uma malha ("grid") de 7,2 x 7,2 m, com carreador de 2,4 m em uma das direções, correspondendo a uma área útil total de 1,33 ha. A área experimental ainda foi subdividida para fins de tratamento localizado, caracterizando a prática de agricultura de precisão em: 128 parcelas com manejo químico localizado (CML ) e 128 parcel as sem manejo quími colocalizado (SML) (Souza et al., 1999).

A resistência mecânica do solo à penetração foi determinada por um penetrógrafo de haste, com ângulo de cone de 30 o, diâmetro da base de cone de $0,01283 \mathrm{~m}$, diâmetro da haste de 0,00953 $\mathrm{m}$ e comprimento de $0,6 \mathrm{~m}$, prescrita pela ASAE S313.2/ 1982, que fornece resultados da força por unidade deárea. Foram real izadas quatro repetições em cada parcela experimental, deforma al eatória. O próximo passo foi tabular os cartões nas camadas de $0-0,1$, 0,1-0,2 e 0,2-0,3 m. Para caracterizar a resistência mecânica do sol o à penetração, as determinações foram realizadas nos meses de abril decada ano da pesquisa.

Para analisar os dados, realizou-se, primeiramente, uma análise exploratória, com intuito de descrever os parâmetros estatísticos os quais ajudam a identificar tendência, dispersão eforma de distribuição dos dados (homogenei dadee normalidade). Na etapa da análise da variabilidade espacial, os dados foram analisados, utilizando-se técnicas de geoestatística, com o intuito de identificar e definir a estrutura de dependência espacial dos dados. Para analisar a variabilidadeespacial, foi utilizado osemivariograma. Para cada variável foram efetuados os cálculos da função semivariância e o ajuste dos model os teóricos a elas. Os val ores das semivariâncias experimentais foram obtidos pelo estimador de Matheron.

Para efetuar o escal onamento dos semivariogramas da variável em estudo, nos três diferentes anos de amostragem, verificou-se a existência de proporcionalidade entre as médias e as variâncias dos dados em estudo. O escal onamento dos semivariogramas foi feito, usando-se a variância dos dados em estudo como fator de escala para agrupar os semivariogramas, conforme Guimarães (1993). Gerou-se, então, um único modelo escal onado para as variáveis estudadas durante o período de três anos, nas três diferentes camadas avaliadas.

O escalonamento dos semivariogramas foi realizado utilizando-se a equação 1.

$$
\gamma_{e s c}=\frac{\gamma_{i}(h)}{\alpha_{i}},(i=1,2, \ldots, \mathrm{s})
$$

sendo $\gamma_{\text {esc }}$ o semivariograma escal onado; $\gamma_{i}(h)$ a semivariância do semivariograma deMatheron, para processos estocásticos gaussianos, intrinsicamente estacionários; s o número de períodos ou camadas medidas; $\alpha_{i}$ ofator de escal onamento, que foi adotado como sendo o valor da respectiva variância dos dados em estudo.

O índice de melhor ajuste "indicative goodness of fit", conforme Pannatier, 1996) foi utilizado na escol ha do mel hor model oajustado aos semivariogramas escal onados, encontrando-se os valores para o efeito pepita $C_{0}$, patamar $C_{0}+C_{1}$, alcance $a$, e coeficiente de efeito pepita $E$. Os parâmentros $C_{0}$, $\mathrm{C}_{0}+\mathrm{C}_{1}$ e a foram, então, utilizados na interpolação dos valores das variáveis em estudo nas posições não amostradas, utilizando-se a técnica de krigagem ordinária. Os mapas de contorno das variáveis estudadas foram final mente confeccionados, usandoseo softwareSURFER 6.0 (Golden Software, 1997).

\section{RESULTADOS E DISCUSSÃO}

No quadro 1, está apresentada a análise estatística dos dados da resistência mecânica do solo à penetração (RMSP), para os anos de 1999 a 2001, 
sob duas formas de manejo, com manejo químico localizado (CML) e sem manejo químico localizado (SML), para as camadas de 0-0, 1, 0,1-0,2 e 0,2-0,3 m. Verificou-se homogeneidade nas parcelas CML e SML para os três anos da pesquisa, considerando que os coeficientes de variação ficaram abaixo de $25 \%$. Os val ores das médias da RMSP de 1999, para os dois tipos de manejo, foram menores na camada de 0,2-0,3 m de camada, em relação às outras camadas analisadas para o ano de 1999. A amplitude dos valores variou de 3,35 MPa, para a camada de 0-0,1 m nas parcelas CML , a 2,41 MPa, para a camada de $0,2-0,3$ m nas parcelas $C M L$. Os altos valores de RMSP encontrados para a camada superficial noano de 1999 podem ser atribuídos ao tráfego intenso de máquinas na área ao longo do primeiro ano da pesquisa, onde foram feitos os tratos culturais das culturas de inverno (trigo) e de verão (soja).

A análise das médias da RMSP de 2000 indicou, para os dois tipos de manejo (CML e SML), valores de amplitude baixa e que, nas três camadas estudadas, não diferiram muito entresi, pois o maior valor foi de 2,54 MPa, para a camada de 0,1-0,2 m nas parcelas CML, e o menor, de 2,38 MPa, para a camada de $0-0,1 \mathrm{~m}$ nas parcelas SML. Analisando os valores médios para a RMSP no ano de 2001 (Quadro 1), observa-se que os val ores obtidos estão em torno de 2,23 a 2,80 MPa, tendo os valores aumentado segundo a camada analisada.
Os valores médios da RMSP encontrados para as diferentes camadas avaliadas nos três anos da pesquisa (Quadro 1) estão dentro dos limites especificados por Torres \& Saraiva (1999), indicando que a RMSP não está sendo um fator restritivo ao crescimento radicular das plantas.

Testes estatísticos comprovaram que todas as variáveis analisadas apresentaram normalidade a $5 \%$ de significância. Com a construção dos semivariogramas direcionais $(0,45,90$ e 135 9), analisou-se a condição de isotropia das variáveis (padrão da estrutura de dependência espacial de todas as variáveis é o mesmo em todas as direções); assim, verificou-se queas variáveis de RMSP doano de 2000, nas camadas de 0-0,1 e 0,1-0,2 m nas parcelas CML eSML, não satisfazem a hipótese de estacionaridade dos dados. Para estas variáveis, utilizou-se a técnica de eliminar a tendência para assegurar que os dados analisados mostrem estacionaridade (utilização dos resíduos).

Os parâmetros e os model os dos semi variogramas escal onados para a variável RMSP nos anos de 1999 a 2001 são apresentados no quadro 2. Observa-se que os model os ajustados foram o model o exponencial, para as variáveis de RMSP, avaliadas nas camadas de0-0,1 e0,2-0,3 m, eo modelo esférico, para a variável deRMSP, avaliada na camada de0,1-0,2 m. A variável RMSP apresentou em todas as camadas uma

Quadro 1. Estatística descritiva da variável resistência mecânica do solo à penetração (RMSP) nos anos de 1999 a 2001, com manejo localizado (CML) e sem manejo localizado (SML)

\begin{tabular}{|c|c|c|c|c|c|c|c|}
\hline & \multirow{2}{*}{ Variável } & \multicolumn{3}{|c|}{ CML } & \multicolumn{3}{|c|}{ SML } \\
\hline & & RMSP $0-0,1$ & RMSP 0,1-0,2 & RMSP 0,2-0,3 & RMSP $0-0,1$ & RMSP 0,1-0,2 & RMSP $0,2-0,3$ \\
\hline \multirow[t]{2}{*}{1999} & $\begin{array}{l}\text { No de amostras } \\
\text { Mínimo (MPa) } \\
\text { Mediana (MPa) } \\
\text { Máximo (MPa) } \\
\text { Média (MPa) } \\
\text { Variância (MPa) }\end{array}$ & $\begin{array}{r}128 \\
1,72 \\
3,20 \\
5,44 \\
3,35 \\
0,53\end{array}$ & $\begin{array}{r}128 \\
1,47 \\
2,55 \\
3,63 \\
2,58 \\
0,10\end{array}$ & $\begin{array}{r}128 \\
1,37 \\
2,45 \\
3,48 \\
2,41 \\
0,07\end{array}$ & $\begin{array}{r}128 \\
1,84 \\
3,23 \\
5,24 \\
3,34 \\
0,44\end{array}$ & $\begin{array}{r}128 \\
1,72 \\
2,60 \\
3,48 \\
2,61 \\
0,10\end{array}$ & $\begin{array}{r}128 \\
1,57 \\
2,47 \\
3,14 \\
2,44 \\
0,08\end{array}$ \\
\hline & C.V. (\%) & 21 & 12 & 11 & 20 & 12 & 11 \\
\hline \multirow[t]{2}{*}{2000} & $\begin{array}{l}\text { No de amostras } \\
\text { Mínimo (MPa) } \\
\text { Mediana (MPa) } \\
\text { Máximo (MPa) } \\
\text { Média (MPa) } \\
\text { Variância (MPa) }\end{array}$ & $\begin{array}{r}128 \\
1,35 \\
2,43 \\
3,31 \\
2,42 \\
0,12\end{array}$ & $\begin{array}{r}128 \\
1,84 \\
2,51 \\
3,40 \\
2,54 \\
0,11\end{array}$ & $\begin{array}{r}128 \\
1,21 \\
2,45 \\
3,37 \\
2,47 \\
0,13\end{array}$ & $\begin{array}{r}128 \\
1,21 \\
2,40 \\
3,31 \\
2,38 \\
0,13\end{array}$ & $\begin{array}{r}128 \\
1,71 \\
2,56 \\
3,78 \\
2,53 \\
0,14\end{array}$ & $\begin{array}{r}128 \\
1,62 \\
2,40 \\
3,51 \\
2,44 \\
0,14\end{array}$ \\
\hline & C.V. (\%) & 14 & 13 & 15 & 15 & 14 & 15 \\
\hline \multirow[t]{2}{*}{2001} & $\begin{array}{l}\text { No de amostras } \\
\text { Mínimo (MPa) } \\
\text { Mediana (MPa) } \\
\text { Máximo (MPa) } \\
\text { Média (MPa) } \\
\text { Variância (MPa) }\end{array}$ & $\begin{array}{r}127 \\
1,51 \\
2,19 \\
3,20 \\
2,23 \\
0,11\end{array}$ & $\begin{array}{r}127 \\
1,55 \\
2,57 \\
3,33 \\
2,59 \\
0,07\end{array}$ & $\begin{array}{r}127 \\
2,05 \\
2,79 \\
3,52 \\
2,80 \\
0,06\end{array}$ & $\begin{array}{r}128 \\
1,17 \\
2,28 \\
3,34 \\
2,28 \\
0,13\end{array}$ & $\begin{array}{r}128 \\
1,82 \\
2,59 \\
3,23 \\
2,61 \\
0,07\end{array}$ & $\begin{array}{r}128 \\
2,27 \\
2,77 \\
3,85 \\
2,80 \\
0,07\end{array}$ \\
\hline & C.V. (\%) & 15 & 10 & 9 & 16 & 10 & 10 \\
\hline
\end{tabular}


dependência espacial moderada, pois os valores do coeficiente de efeito pepita (E) ficaram dentro dos limites de 25 a 75 \% (Cambardella et al., 1994; Souza et al., 1999). Os alcances (a) dos semivariogramas escaIonados nos diferentes anos da variável RMSP foram maiores que $50 \mathrm{~m}$. Destemodo, a autocorrelação entre os pontos amostrados, ou a dependência espacial entre as amostras das variáveis, atingiu mais da metade da distância total considerada do alcance (100 m).

Os gráficos dos semivariogramas escalonados para a variável RMSP nas camadas de $0-0,1,0,1-0,2$ e 0,2-0,3 m são apresentados na figura 1 .

No quadro 3, estão apresentados os model os e os parâmetros ajustados dos semivariogramas

Quadro 2. Parâmetros e modelos dos semivariogramas escalonados para a variável RMSP, nos anos de 1999 a 2001

\begin{tabular}{|c|c|c|c|c|c|c|c|}
\hline Variável & Camada & Modelo & $\mathrm{C}_{0}$ & $\mathbf{C}_{1}$ & $\left(C_{1}+C_{0}\right)$ & $a$ & $E=C_{0} /\left(C_{1}+C_{0}\right)$ \\
\hline & $\mathrm{m}$ & & & & & & $\%$ \\
\hline $\begin{array}{l}C M L \\
\text { SML }\end{array}$ & $0-\quad 0,1$ & $\begin{array}{l}\text { Exponencial } \\
\text { Exponencial }\end{array}$ & $\begin{array}{l}0,4785 \\
0,5423\end{array}$ & $\begin{array}{l}0,6002 \\
0,5246\end{array}$ & $\begin{array}{l}1,0787 \\
1,0669\end{array}$ & $\begin{array}{l}80,52 \\
65,24\end{array}$ & $\begin{array}{l}44 \\
51\end{array}$ \\
\hline $\begin{array}{l}\text { CML } \\
\text { SML }\end{array}$ & $0,1-0,2$ & $\begin{array}{l}\text { Esférico } \\
\text { E sférico }\end{array}$ & $\begin{array}{l}0,6987 \\
0,6742\end{array}$ & $\begin{array}{l}0,3256 \\
0,3895\end{array}$ & $\begin{array}{l}1,0243 \\
1,0637\end{array}$ & $\begin{array}{l}75,63 \\
72,54\end{array}$ & $\begin{array}{l}68 \\
63\end{array}$ \\
\hline $\begin{array}{l}\text { CML } \\
\text { SML }\end{array}$ & $0,2-0,3$ & $\begin{array}{l}\text { Exponencial } \\
\text { Exponencial }\end{array}$ & $\begin{array}{l}0,6325 \\
0,6235\end{array}$ & $\begin{array}{l}0,3845 \\
0,4732\end{array}$ & $\begin{array}{l}1,0170 \\
1,0967\end{array}$ & $\begin{array}{l}61,23 \\
66,32\end{array}$ & $\begin{array}{l}62 \\
57\end{array}$ \\
\hline
\end{tabular}

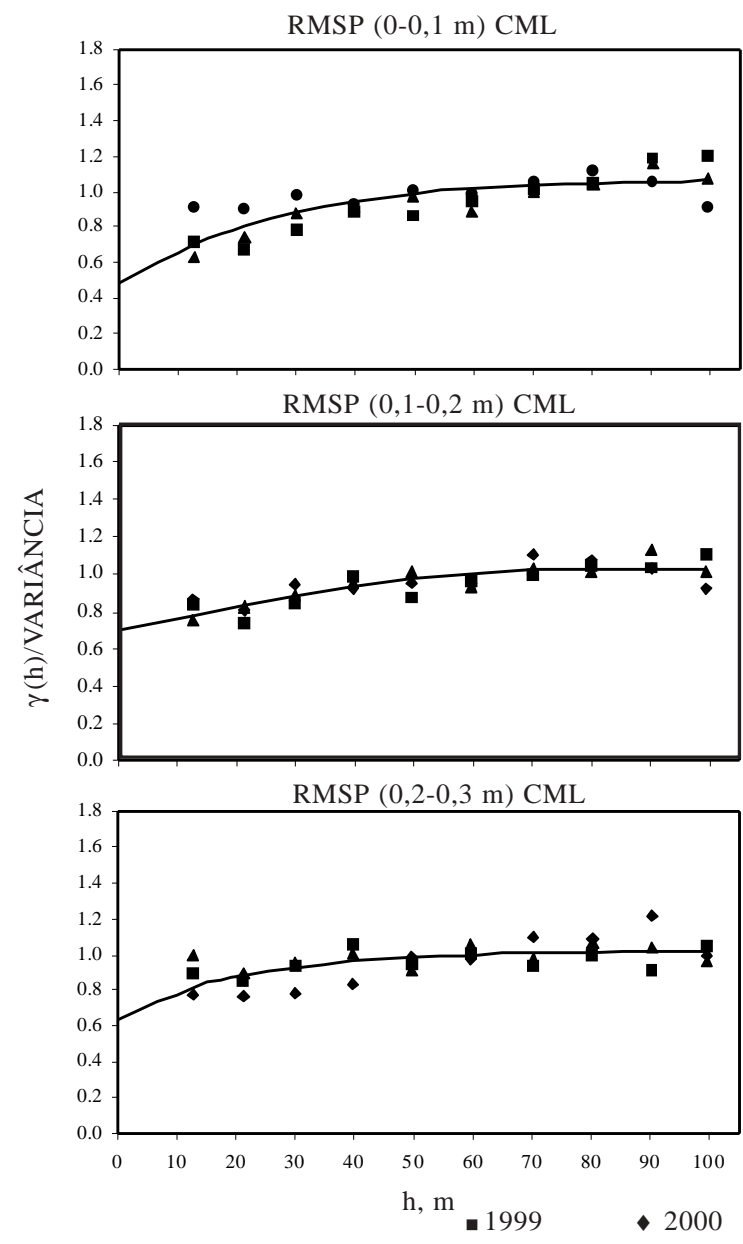

(a)

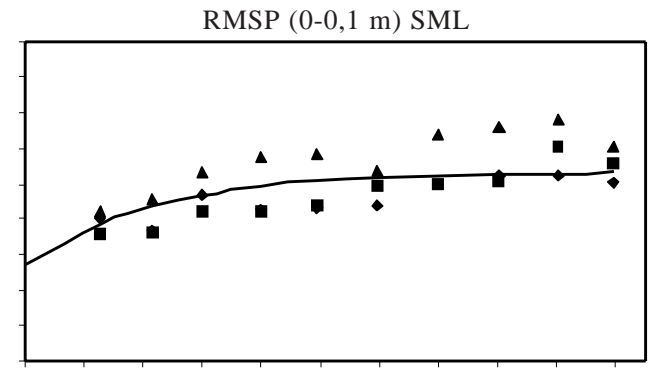

$\operatorname{RMSP}(0,1-0,2 \mathrm{~m}) \mathrm{SML}$

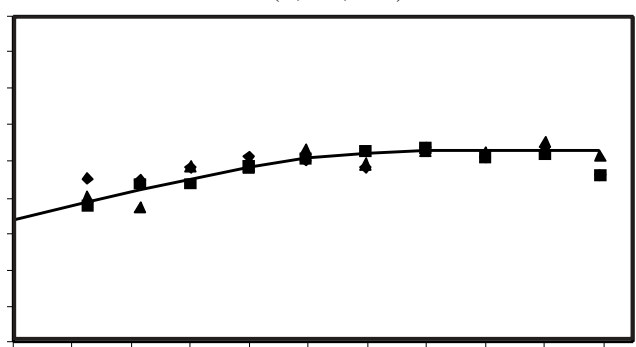

$\operatorname{RMSP}(0,2-0,3 \mathrm{~m}) \mathrm{SML}$

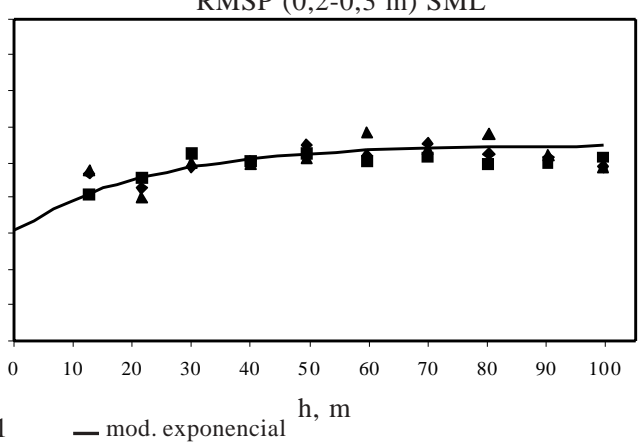

Figura 1. Semivariogramas escalonados para a variável $\operatorname{RMSP}(0-0,1 \mathrm{~m})(\mathrm{a}),(0,1-0,2 \mathrm{~m})(\mathrm{b})$ e(0,2-0,3 m) (c), avaliada nos anos de 1999 a 2001, nas parcelas com e sem manejo químico localizado (CML e SML, respectivamente). 
escalonados para a variável RMSP nas diferentes camadas (0-0,1, 0,1-0,2 e 0,2-0,3 m).

Como pode ser observado no quadro 3 , os model os ajustados aos semivariogramas escalonados foram o modelo esférico, para as variáveis de RMSP dos anos de 1999 e 2000, e o model o exponencial, para a variável deRMSP doanode2001. O grau dedependência espacial, segundo a classificação de Cambardella et al. (1994) e Souza et al . (1999), nas variáveis deRMSP nos anos de 1999 a 2001, apresentou-se como moderado, pois os valores do coeficiente de efeito pepita (E) estão dentro dos limites de 25 a $75 \%$. Os alcances (a) dos semivariogramas escalonados nas diferentes camadas da variável RMSP, para os anos de 1999 a 2001 apresentaram-se novamente maiores que $50 \mathrm{~m}$. Os gráficos dos semivariogramas escalonados para a variável RMSP nos anos de 1999 a 2001, nas diferentes camadas, estão apresentados na figura 2.

Quadro 3. Parâmetros e modelos dos semivariogramas escalonados para a variável R MSP nas camadas de 0-0,1, 0,1-0,2 e 0,2-0,3 m

\begin{tabular}{|c|c|c|c|c|c|c|c|}
\hline Variável & Ano & Modelo & $\mathrm{C}_{0}$ & $C_{1}$ & $\left(C_{1}+C_{0}\right)$ & $a$ & $E=C_{0} /\left(C_{1}+C_{0}\right)$ \\
\hline & & & & & & & $\%$ \\
\hline $\begin{array}{l}\text { CML } \\
\text { SML }\end{array}$ & 1999 & $\begin{array}{l}\text { Esférico } \\
\text { Esférico }\end{array}$ & $\begin{array}{l}0,6023 \\
0,6253\end{array}$ & $\begin{array}{l}0,4132 \\
0,4123\end{array}$ & $\begin{array}{l}1,0155 \\
1,0376\end{array}$ & $\begin{array}{l}72,56 \\
72,35\end{array}$ & $\begin{array}{l}59 \\
60\end{array}$ \\
\hline $\begin{array}{l}\text { CML } \\
\text { SML }\end{array}$ & 2000 & $\begin{array}{l}\text { Esférico } \\
\text { Esférico }\end{array}$ & $\begin{array}{l}0,6835 \\
0,6532\end{array}$ & $\begin{array}{l}0,3954 \\
0,4012\end{array}$ & $\begin{array}{l}1,0789 \\
1,0544\end{array}$ & $\begin{array}{l}78,52 \\
69,53\end{array}$ & $\begin{array}{l}63 \\
62\end{array}$ \\
\hline $\begin{array}{l}\text { CML } \\
\text { SML }\end{array}$ & 2001 & $\begin{array}{l}\text { Exponencial } \\
\text { Exponencial }\end{array}$ & $\begin{array}{l}0,5243 \\
0,6123\end{array}$ & $\begin{array}{l}0,5342 \\
0,5125\end{array}$ & $\begin{array}{l}1,0585 \\
1,1248\end{array}$ & $\begin{array}{l}71,25 \\
71,56\end{array}$ & $\begin{array}{l}50 \\
54\end{array}$ \\
\hline
\end{tabular}

$\mathrm{C}_{0}=$ efeito pepita; $\left(\mathrm{C}_{1}+\mathrm{C}_{0}\right)=$ patamar; $\alpha=$ alcance; $\mathrm{E}=$ coeficiente de efeito pepita.

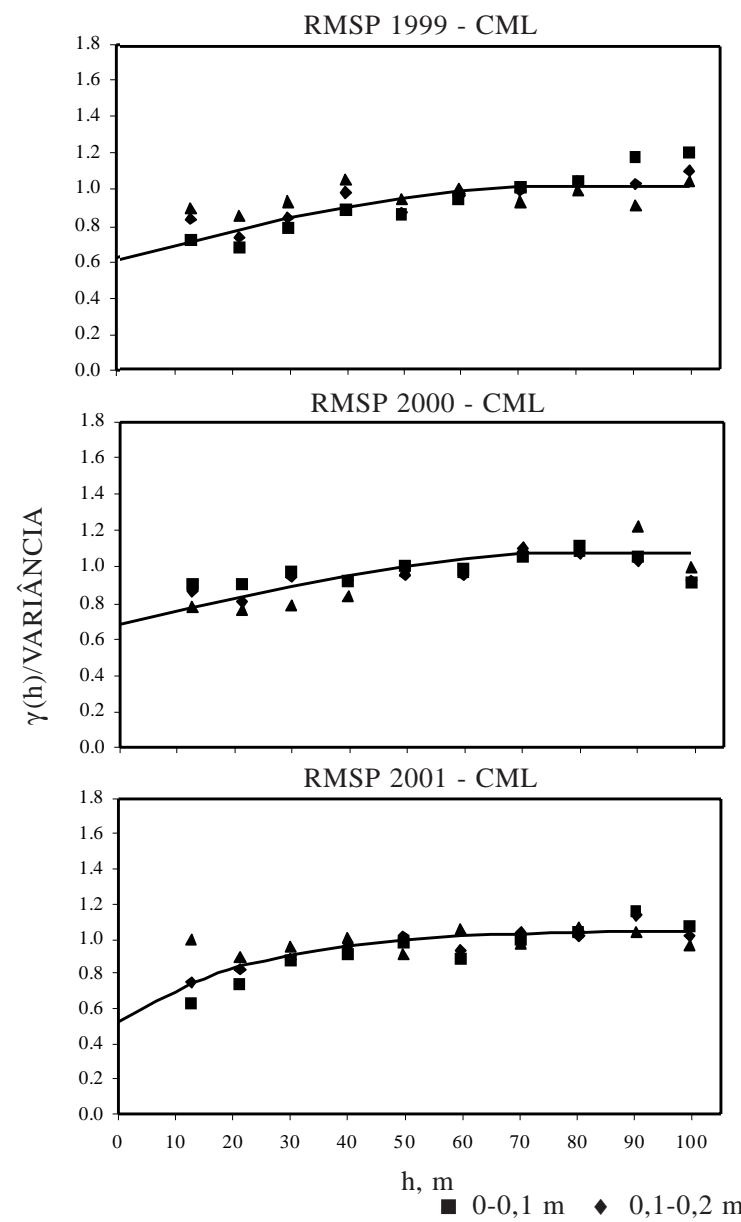

(a)

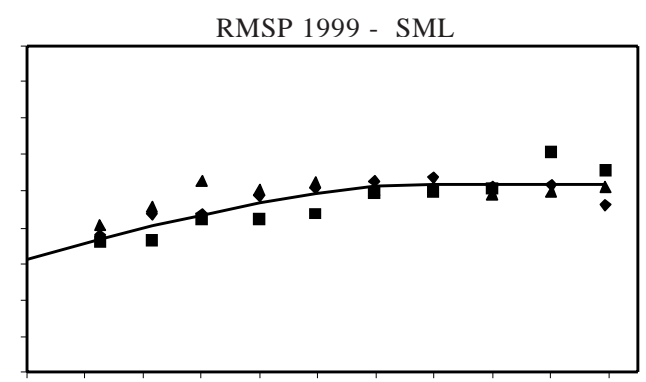

RMSP 2000 - SML

(b)

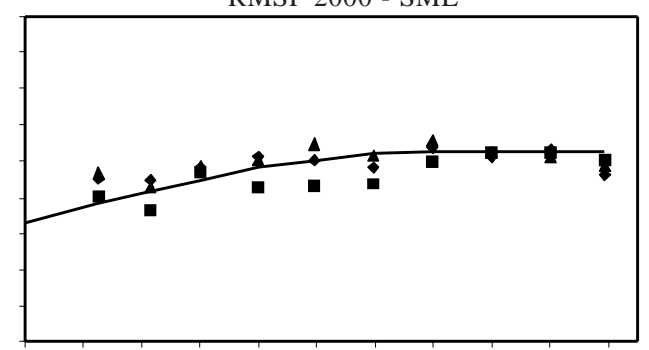

RMSP 2001 - SML

(c)

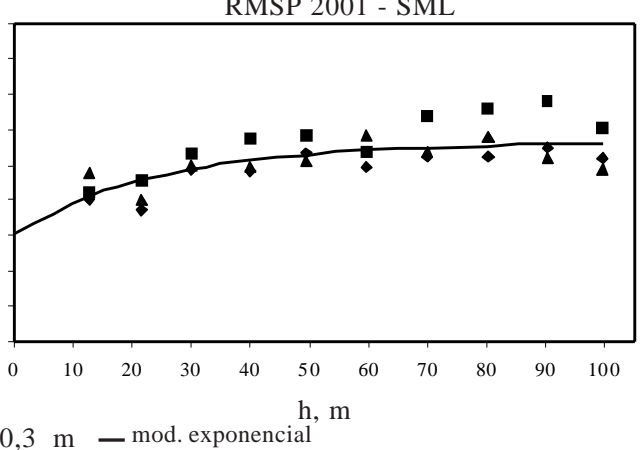

Figura 2. Semivariogramas escalonados para a variável RMSP 1999 (a), 2000 (b) e 2001 (c), avaliada nas camadas de 0-0,1, 0,1-0,2 e 0,2-0,3 m, nas parcelas com e sem manejo quími co localizado (CML e SML, respectivamente). 
Realizou-se também o ajuste dos semivariogramas individuais (Quadro 4), utilizando-se o estimador de Matheron. Os modelos ajustados aos semi variogramas individuais foram o esférico e o exponencial, os valores de al cance (a) e o grau de dependência espacial dos semivariogramas individuais não diferiram muito dos valores encontrados para os semivariogramas escal onados (Quadros 2 e 3).

Com base nos parâmetros de ajuste (efeito pepita $\left(C_{0}\right)$, patamar $\left(C_{0}+C_{1}\right)$, alcance $\left.(a)\right)$ e nos modelos ajustados aos semivariogramas individuais e aos semivariogramas escalonados, descritos anteriormente, foi possível a construção dos mapas de contorno (superfície contínua) para cada variável em estudo, caracterizando, assim, o comportamento espacial de cada variável no campo. Os mapas de contorno da RMSP foram construídos, adotando, como escala de classificação, a média dos valores mínimos e dos máximos obtidos nos três anos da pesquisa nas parcelas CML e SML; desta forma, a escala de valores dos mapas de RMSP apresentou uma amplitude de 1,50 a 3,30 MPa.

As figuras 3 a 5 mostram os mapas de contorno da variável RMSP dos anos de 1999 (RMSP 99), 2000 (RMSP 00) e 2001 (RMSP 01), construídos a partir dos parâmetros dos semivariogramas individuais e dos semivariogramas escal onados. Os gráficos são discretizados de acordo com o tipo de mobilização do solo (CML e SML ) e da camada anal isada.

Percebe-se que os mapas da camada superficial (0-0,1 m) apresentam valores com amplitudede2,70 a 3,30 MPa; da camada intermediária (0,1-0,2 m), valores de 2,10 a 2,70 MPa, eda camada inferior (0,2$0,3 \mathrm{~m}$ ), valores de 1,70 a 2,50 MPa (Figura 3). Estes valores são considerados baixos para a cultura da soja estabelecida em Latossolo Bruno (Torres \& Saraiva, 1999).

Verifica-se que os mapas da camada superficial (0-0,1 m) e intermediária (0,1-0,2 m) mostram valores com amplitude de 2,10 a 2,70 MPa, respectivamente, e da camada inferior $(0,2-0,3 \mathrm{~m})$, valores de 1,90 a 2,50 MPa (Figura 4). Desta forma, pode-sedizer quea área experimental no ano de 2000 não apresentou valores de RMSP que fossem restritivos ao crescimento radicular ou indicativos de alta compactação em todas as camadas avaliadas.

Verifica-se que os mapas da camada superficial (0-0,1 m) mostram valores com amplitude de 1,70 a 2,90 MPa; da camada intermediária (0,1-0,2 m), valores de 2,10 a 2,70 MPa e, da camada inferior $(0,2-$ $0,3 \mathrm{~m}$ ), val ores de 2,50 a 3,10 MPa (Figura 5). Assim, pode-se dizer novamente que a área experimental no ano de 2001 também não apresentou valores de RMSP restritivos ao crescimento radicular e indicativos deal ta compactação em todas as camadas avaliadas.

Pode-se notar também (Figuras de 3 a 5) que os métodos de manejo utilizados não causaram grandes diferenças entre os mapas, ou seja, os mapas construídos a partir dos dados das parcelas CML revelam comportamento espacial parecido com os mapas construídos a partir dos dados das parcelas SML. Entretanto, quando se comparam os mapas de RMSP para as diferentes camadas de análise $0-0,1,0,1-0,2$ e 0,2-0,3 m, e em cada ano de

Quadro 4. Parâmetros e modelos dos semivariogramas individuais para a variável RMSP, nos anos de 1999 a 2001, nas camadas de 0-0,1, 0,1-0,2 e 0,2-0,3 m

\begin{tabular}{|c|c|c|c|c|c|c|c|c|}
\hline Ano & Variável & Camada & Modelo & $C_{0}$ & $C_{1}$ & $\left(C_{1}+C_{0}\right)$ & $a$ & $E=C_{0} /\left(C_{1}+C_{0}\right)$ \\
\hline & & $\mathrm{m}$ & & & & & & $\%$ \\
\hline \multirow{2}{*}{1999} & CML & $\begin{array}{l}0-0,1 \\
0,1-0,2 \\
0,2-0,3\end{array}$ & $\begin{array}{l}\text { Esférico } \\
\text { Exponencial } \\
\text { Exponencial }\end{array}$ & $\begin{array}{l}0,2640 \\
0,0579 \\
0,0520\end{array}$ & $\begin{array}{l}0,3240 \\
0,0500 \\
0,0240\end{array}$ & $\begin{array}{l}0,5880 \\
0,1079 \\
0,0760\end{array}$ & $\begin{array}{l}91,31 \\
83,69 \\
72,00\end{array}$ & $\begin{array}{l}44,89 \\
53,66 \\
68,42\end{array}$ \\
\hline & SML & $\begin{array}{l}0-0,1 \\
0,1-0,2 \\
0,2-0,3\end{array}$ & $\begin{array}{l}\text { Esférico } \\
\text { Exponencial } \\
\text { Esférico }\end{array}$ & $\begin{array}{l}0,2450 \\
0,0560 \\
0,0544\end{array}$ & $\begin{array}{l}0,2250 \\
0,0540 \\
0,0272\end{array}$ & $\begin{array}{l}0,4700 \\
0,1100 \\
0,0816\end{array}$ & $\begin{array}{l}86,69 \\
81,00 \\
51,00\end{array}$ & $\begin{array}{l}52,12 \\
50,90 \\
66,66\end{array}$ \\
\hline \multirow{2}{*}{2000} & CML & $\begin{array}{l}0-0,1 \\
0,1-0,2 \\
0,2-0,3\end{array}$ & $\begin{array}{l}\text { Exponencial } \\
\text { Exponencial } \\
\text { Esférico }\end{array}$ & $\begin{array}{l}0,0760 \\
0,0399 \\
0,0760\end{array}$ & $\begin{array}{l}0,0360 \\
0,0287 \\
0,0600\end{array}$ & $\begin{array}{l}0,1120 \\
0,0686 \\
0,1360\end{array}$ & $\begin{array}{l}88,00 \\
85,00 \\
79,00\end{array}$ & $\begin{array}{l}67,85 \\
58,16 \\
55,88\end{array}$ \\
\hline & SML & $\begin{array}{l}0-0,1 \\
0,1-0,2 \\
0,2-0,3\end{array}$ & $\begin{array}{l}\text { Exponencial } \\
\text { Exponencial } \\
\text { Exponencial }\end{array}$ & $\begin{array}{l}0,0530 \\
0,0512 \\
0,0940\end{array}$ & $\begin{array}{l}0,0440 \\
0,0256 \\
0,0540\end{array}$ & $\begin{array}{l}0,0970 \\
0,0768 \\
0,1480\end{array}$ & $\begin{array}{l}80,00 \\
75,00 \\
59,00\end{array}$ & $\begin{array}{l}54,64 \\
66,66 \\
63,51\end{array}$ \\
\hline \multirow{2}{*}{2001} & CML & $\begin{array}{l}0-0,1 \\
0,1-0,2 \\
0,2-0,3\end{array}$ & $\begin{array}{l}\text { Exponencial } \\
\text { Exponencial } \\
\text { Exponencial }\end{array}$ & $\begin{array}{l}0,0500 \\
0,0371 \\
0,0476\end{array}$ & $\begin{array}{l}0,0660 \\
0,0308 \\
0,0154\end{array}$ & $\begin{array}{l}0,1160 \\
0,0679 \\
0,0630\end{array}$ & $\begin{array}{l}78,00 \\
68,00 \\
57,00\end{array}$ & $\begin{array}{l}43,10 \\
54,63 \\
75,55\end{array}$ \\
\hline & SML & $\begin{array}{l}0-0,1 \\
0,1-0,2 \\
0,2-0,3\end{array}$ & $\begin{array}{l}\text { Exponencial } \\
\text { Exponencial } \\
\text { Exponencial }\end{array}$ & $\begin{array}{l}0,0760 \\
0,0392 \\
0,0480\end{array}$ & $\begin{array}{l}0,0620 \\
0,0301 \\
0,0312\end{array}$ & $\begin{array}{l}0,1380 \\
0,0693 \\
0,0792\end{array}$ & $\begin{array}{l}62,00 \\
60,06 \\
74,00\end{array}$ & $\begin{array}{l}55,07 \\
56,56 \\
60,60\end{array}$ \\
\hline
\end{tabular}

$\mathrm{C}_{0}=$ efeito pepita; $\left(\mathrm{C}_{1}+\mathrm{C}_{0}\right)$ = patamar; $\alpha=$ alcance; $\mathrm{E}=$ coeficiente de efeito pepita. 


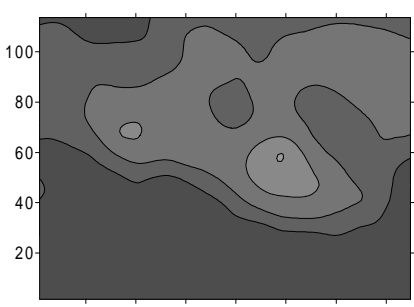

RMSP 99 (0-0,1 m) - CML

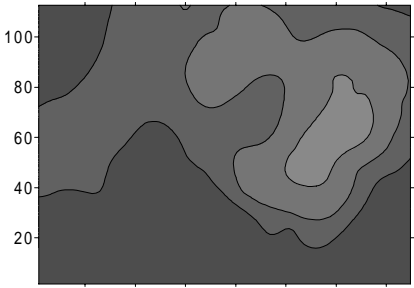

RMSP 99 (0-0,1 m) - SML

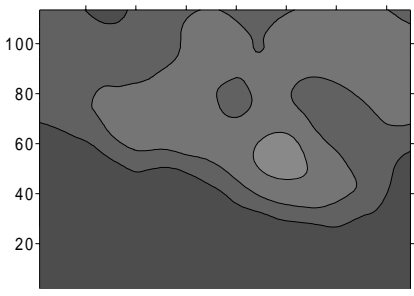

RMSP 99 (0-0,1 m) - CML

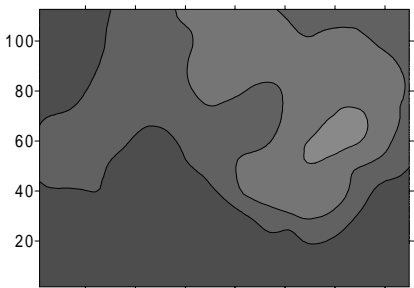

RMSP 99 (0-0,1 m) - SML

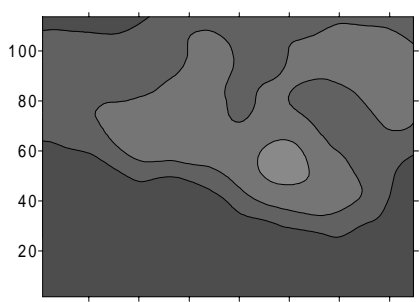

RMSP 99 (0-0,1 m) - CML

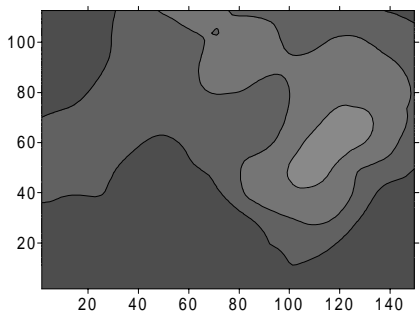

RMSP 99 (0-0,1 m) - SML

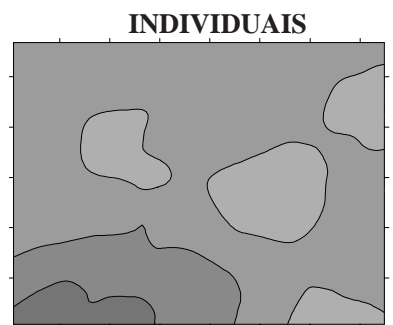

RMSP 99 (0,1-0,2 m) - CML

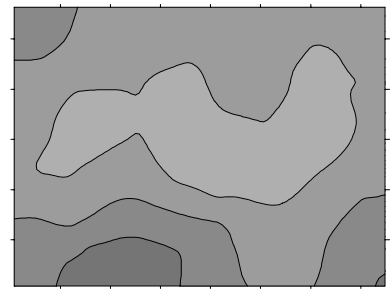

RMSP 99 (0,1-0,2 m) - SML

\section{ESCALONADOS NOS DIFERENTES ANOS}

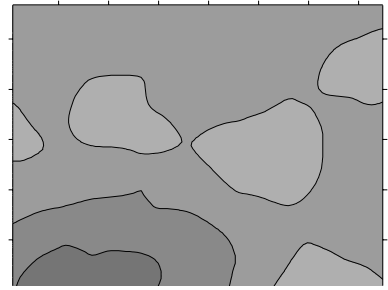

RMSP 99 (0,1-0,2 m) - CML

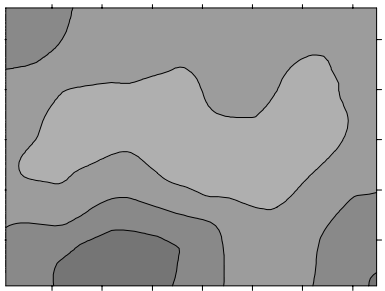

RMSP 99 (0,1-0,2 m) - SML

\section{ESCALONADOS NAS DIFERENTES CAMADAS}

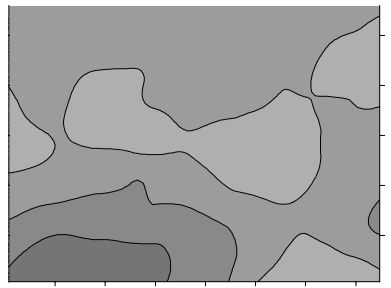

RMSP 99 (0,1-0,2 m) - CML

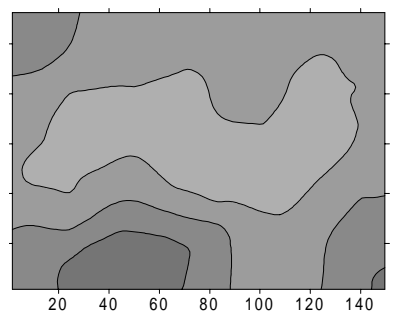

RMSP 99 (0,1-0,2 m) - SML
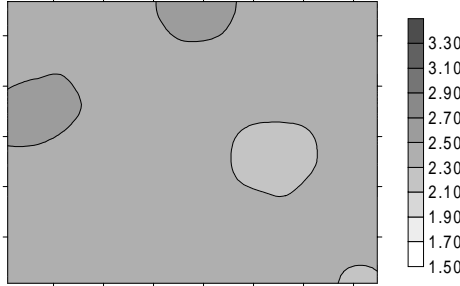

RMSP 99 (0,2-0,3 m) - CML

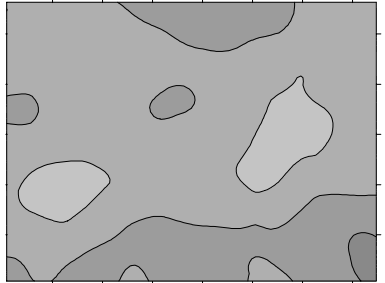

RMSP 99 (0,2-0,3 m) - SML

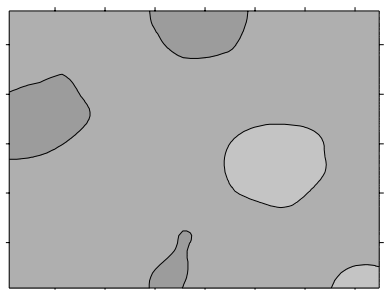

RMSP 99 (0,2-0,3 m) - CML

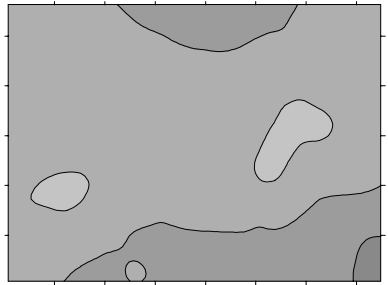

RMSP 99 (0,2-0,3 m) - SML

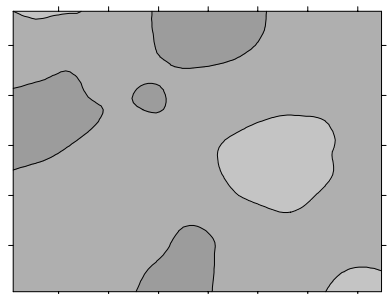

RMSP $99(0,2-0,3$ m) - CML

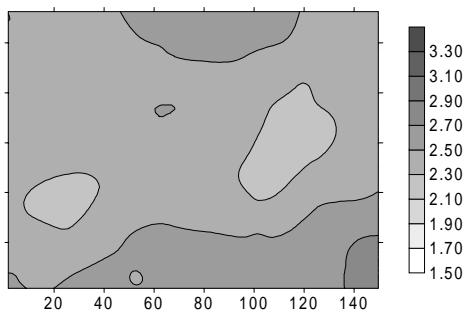

RMSP 99 (0,2-0,3 m) - SML

Figura 3. Mapas de contorno da variável RMSP (MPa) do ano de 1999, construídos a partir dos semivariogramas individuais; escalonados nos diferentes anos de amostragem e nas diferentes camadas. 


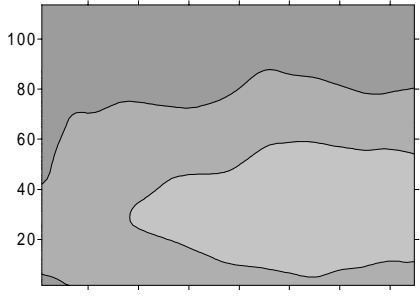

RMSP $00(0-0,1 \mathrm{~m})$ - CML

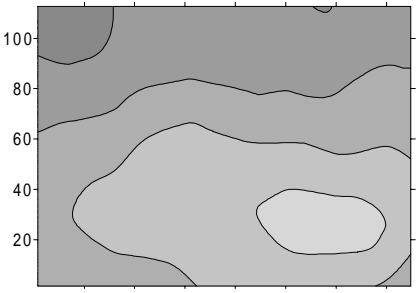

RMSP $00(0-0,1 \mathrm{~m})$ - SML

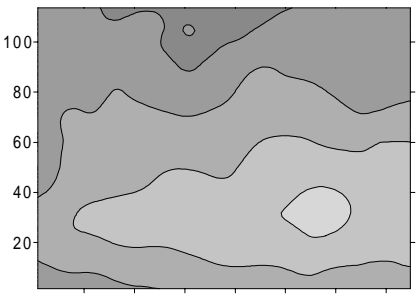

RMSP $00(0-0,1 \mathrm{~m})$ - CML

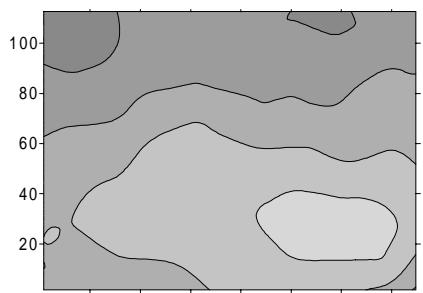

RMSP $00(0-0,1 \mathrm{~m})$ - SML

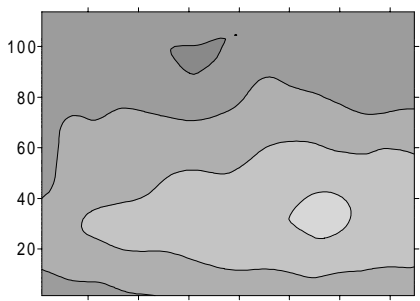

RMSP $00(0-0,1 \mathrm{~m})-\mathrm{CML}$

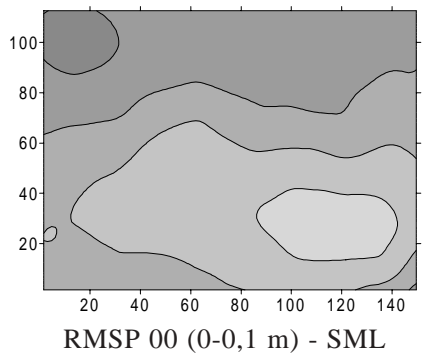

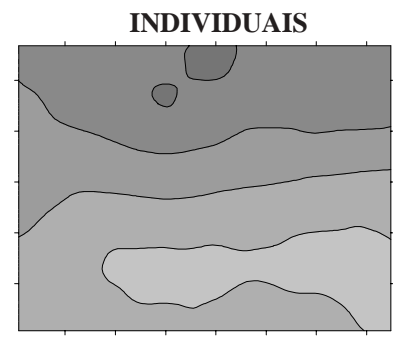
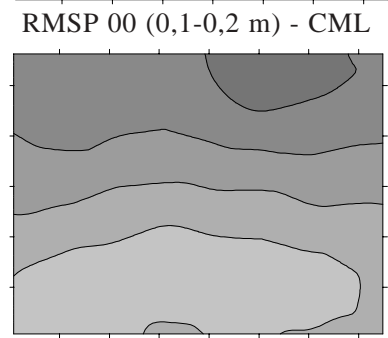

RMSP $00(0,1-0,2 \mathrm{~m})-\mathrm{SML}$

\section{ESCALONADOS NOS DIFERENTES ANOS}

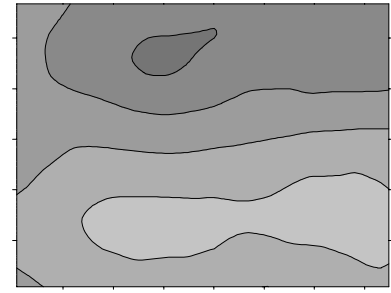

RMSP $00(0,1-0,2 \mathrm{~m})-\mathrm{CML}$

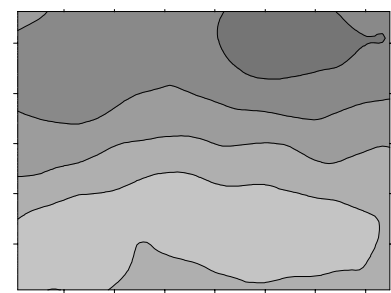

RMSP $00(0,1-0,2 \mathrm{~m})$ - SML

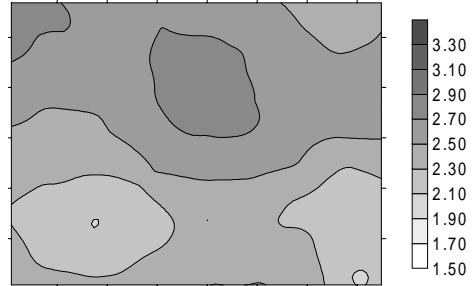

RMSP $00(0,2-0,3 \mathrm{~m})-\mathrm{CML}$

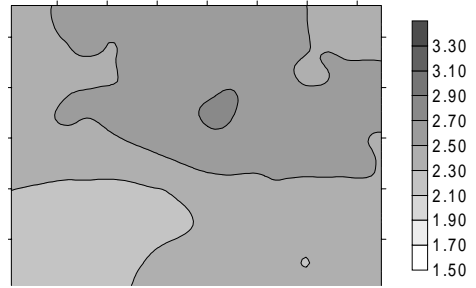

RMSP $00(0,2-0,3 \mathrm{~m})-\mathrm{SML}$

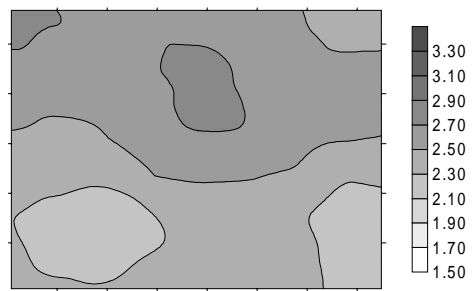

RMSP $00(0,2-0,3 \mathrm{~m})-\mathrm{CML}$

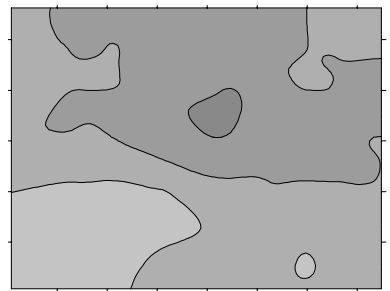

RMSP $00(0,2-0,3 \mathrm{~m})-\mathrm{SML}$

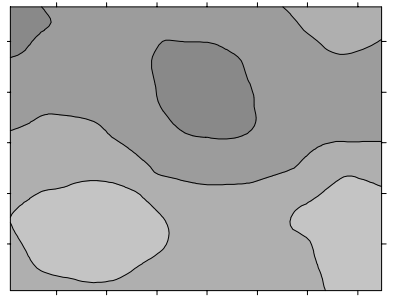

RMSP $00(0,2-0,3 \mathrm{~m})-\mathrm{CML}$

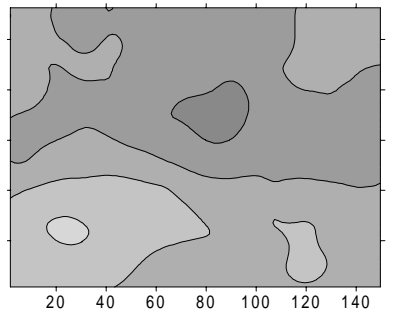

RMSP 00 (0,2-0,3 m) - SML

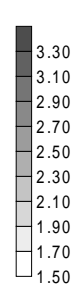

1.50
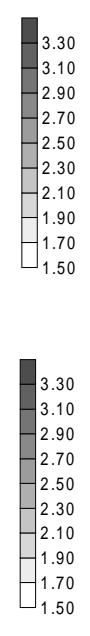

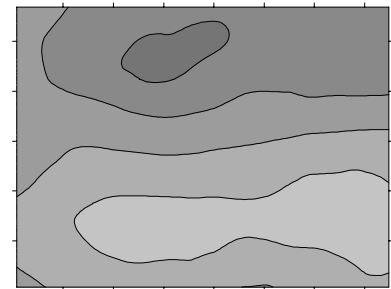

RMSP $00(0,1-0,2 \mathrm{~m})$ - CML

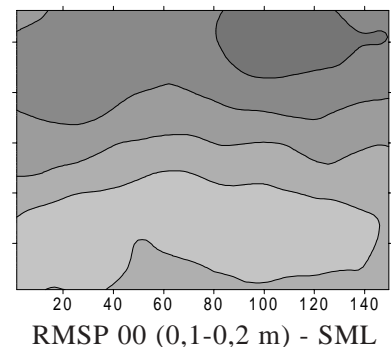

RMSP $00(0,1-0,2 \mathrm{~m})$ - SML

\section{ESCALONADOS NAS DIFERENTES CAMADAS}

Figura 4. Mapas de contorno da variável RMSP (MPa) do ano de 2000, construídos a partir dos semivariogramas individuais; escalonados nos diferentes anos de amostragem e nas diferentes camadas. 


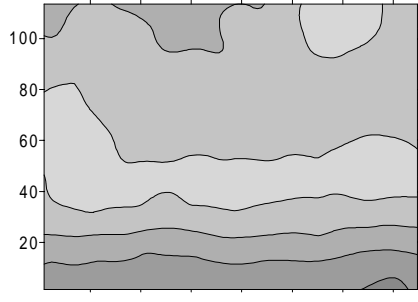

RMSP $01(0-0,1 \mathrm{~m})$ - CML

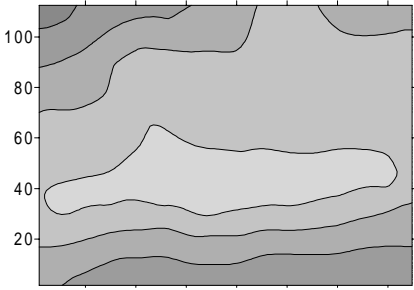

RMSP $01(0-0,1 \mathrm{~m})$ - SML

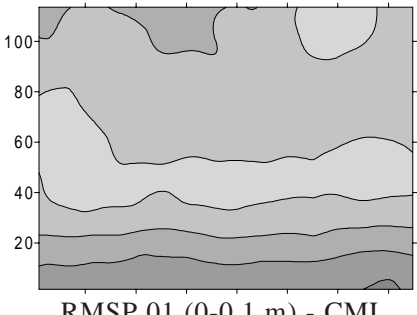

$01(0-0,1 \mathrm{~m})-\mathrm{CML}$

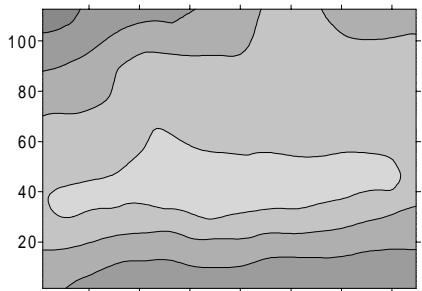

RMSP $01(0-0,1 \mathrm{~m})$ - SML

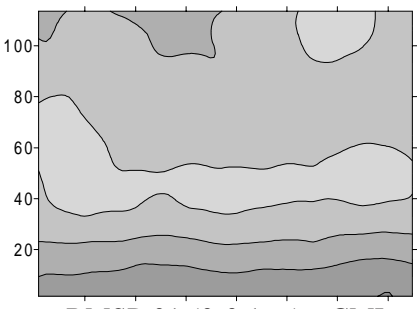

RMSP $01(0-0,1 \mathrm{~m})$ - CML

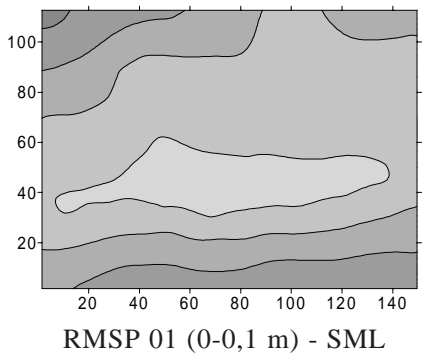

INDIVIDUAIS

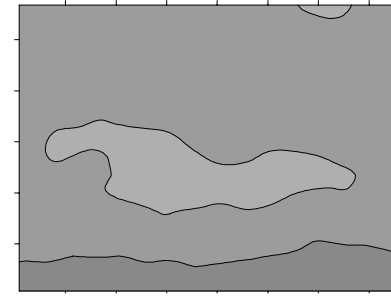

RMSP 01 (0,1-0,2 m) - CML

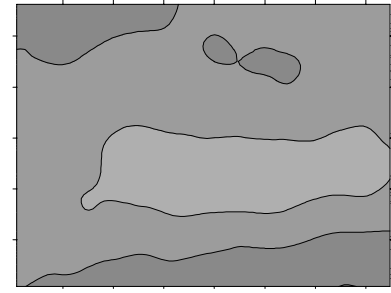

RMSP $01(0,1-0,2 \mathrm{~m})$ - SML

\section{ESCALONADOS NOS DIFERENTES ANOS}

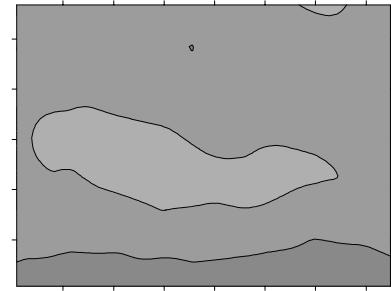

RMSP 01 (0,1-0,2 m) - CML

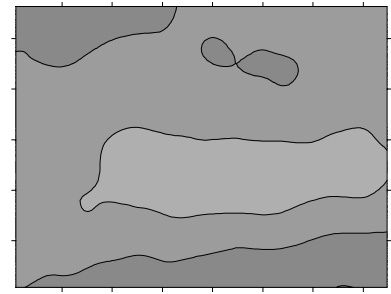

RMSP 01 (0,1-0,2 m) - SML

\section{ESCALONADOS NAS DIFERENTES CAMADAS}

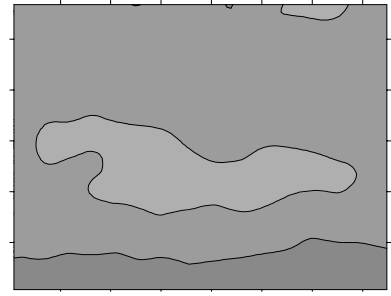

RMSP $01(0,1-0,2$ m) - CML

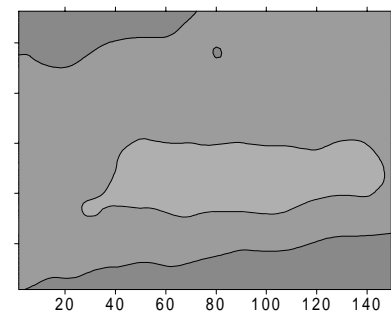

RMSP 01 (0,1-0,2 m) - SML

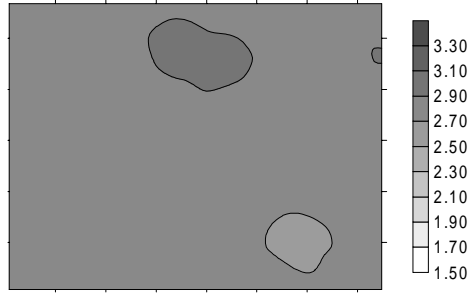

RMSP $01(0,2-0,3$ m) - CML

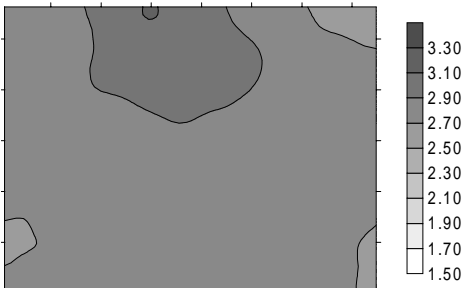

RMSP $01(0,2-0,3$ m) - SML

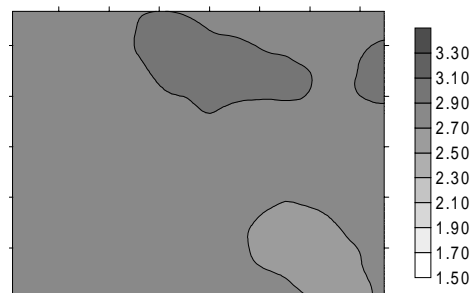

RMSP $01(0,2-0,3 \mathrm{~m})$ - CML

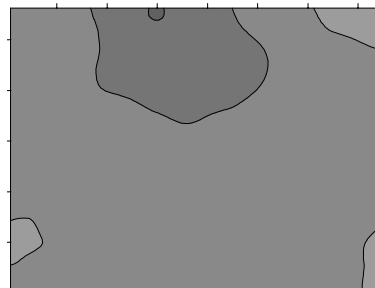

RMSP $01(0,2-0,3 \mathrm{~m})$ - SML

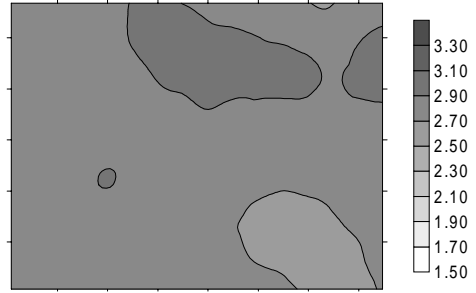

RMSP $01(0,2-0,3$ m) - CML

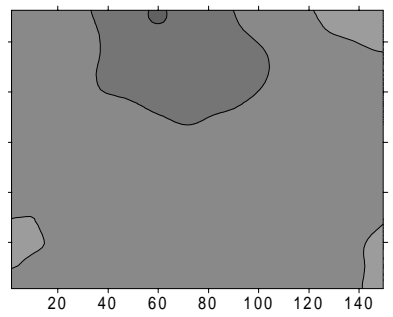

RMSP 01 (0,2-0,3 m) - SML

Figura 5. Mapas de contorno da variável RMSP (MPa) do ano de 2001, construídos a partir dos semivariogramas individuais; escalonados nos diferentes anos de amostragem e nas diferentes camadas. 
amostragem (1999, 2000 e 2001), percebe-se diferenças no comportamento da variabilidade espacial entre eles.

Analisando a variabilidade temporal da variável RMSP, pode-se perceber, nos mapas de contorno ( $F$ iguras de 3 a 5), que o comportamento e a amplitude da variabilidade foram diferentes na área experimental aolongo dos anos (1999, 2000 e 2001), tanto nas parcelas CML quanto nas parcelas SML.

Verificou-se, ainda, que não houve diferença entre os mapas de contorno da variável RMSP gerados a partir dos parâmetros de ajuste dos semivariogramas individuais e os mapas de contorno construídos a partir dos dois métodos de ajustes dos semivario-gramas escal onados (anualmente e em diferentes camadas).

\section{CONCLUSÕES}

1. A variável resistência mecânica do solo à penetração (RMSP) apresentou variabilidade espacial com comportamento distinto, conforme a camada e o ano de estudo.

2. A variável RMSP apresentou variabilidade temporal tanto nas parcelas com manejo químico localizado (CML) quanto nas parcelas sem manejo químico localizado (SML).

3. As duas formas de manejo (CML) e (SML) não influenciaram o comportamento espacial da variável RMSP.

4. O escalonamento dos semivariogramas reduziu o tempo computacional de ajuste dos modelos, não apresentando diferenças no comportamento e na amplitude da variabilidade espacial em relação aos semivariogramas não escalonados.

\section{AGRADE CIMENTOS}

À CAPES, CNPq ea Fundação Araucária (PR), pelo apoio financeiro concedido. Aos membros do Núcleo de Inovações Tecnológicas (NIT), da Universidade Estadual do Oeste do Paraná (UNIOESTE), e aos membros da Cooperativa Central Agropecuária de Desenvolvimento Tecnológico e E conômico Ltda (COODETEC), pela ajuda concedida ao desenvolvimento da pesquisa.

\section{LITERATURA CITADA}

CAMBARDELLA, C.A.; MOORMAM, T.B.; NOVAK, J .M.; PARKIN, T.B.; KARLEN, D.L.; TURCO, R.F.\& KONOPKA, A.E. Field-scale variability of soil properties in central lowa soils. Soil Sci. Soc. Am. J ., 58:1501-1511, 1994.

CASCAVEL. Prefeitura Municipal. Proposta para recuperação ambiental da bacia hidrográfica do Rio Cascavel. Cascavel, 1995. 164p.

CORÁ, J .E. \& MARQUES J r., J . Mecanização e Agricultura de Precisão. In: CONGRESSO BRASILEIRO DE ENGENHARIA AGRíCOLA, 27., Poços de Caldas, 1998. Simpósio. Lavras, Universidade Federal de Lavras Atributos do solo para agricultura de precisão, 1998. p.3170.

EMPRESA BRASILEIRA DE PESQUISA AGROPECUÁRIA EMBRAPA. Centro Nacional de Pesquisa de Solos. Sistema Brasileiro de classificação de solos. Brasília, 1999. 412p.

GOLDEM SOFTWARE. Surfer for Windows - User's Guide Release 6.0. USA, 1997. 340p.

GUIMARÃES, E.C. Variabilidade espacial da umidade e da densidade do solo em um Latossolo Roxo. Campinas, Universidade de Campinas, 1993. 138p. (Tese de Mestrado)

KLEIN, V.A.; LIBARDI, P.L. \& SILVA, A.P. Resistência mecânica do solo à penetração sob diferentes condições de densidade e teor de água. Eng. Agríc.,18:45-54, 1998.

MANZATTO, C.V.; BHERING, S.B. \& SIMÕES, M. Agricultura de precisão: propostas eações da Embrapa sol os. Disponível em: \http://www.cnps.embrapa.br/search/pesqs/proj01/ proj01.html > Acesso em: 18 abr. 2000.

PANNATIER, Y. Variowin: software for epatial date analysis in 2D. New York, Springer-Verlag, 1996. 96p.

SOUZA, E.G.; J OHANN, J .A.; ROCHA, J .V.; RIBEIRO, S.R.A.; SILVA, M.S.; URIBE-OPAZO, M.A.; MOLIN, J.P.; OLIVEIRA, E.F. \& NÓBREGA, L.H.P. Variabilidade espacial dos atributos químicos do solo em um Latossolo Roxo distrófico da região de Cascavel-PR. Eng. Agric., 18:80-92, 1999

TORRES, E. \& SARAIVA, O.F. Camadas de impedimento do solo em sistemas agrícolas com a soja. Londrina, Empresa Brasileira de Pesquisa Agropecuária, 1999. 58p. (Circular Técnica, 23) 
E. MERCANTE et al.

R. Bras. Ci. Solo, 27:1149-1159, 2003 